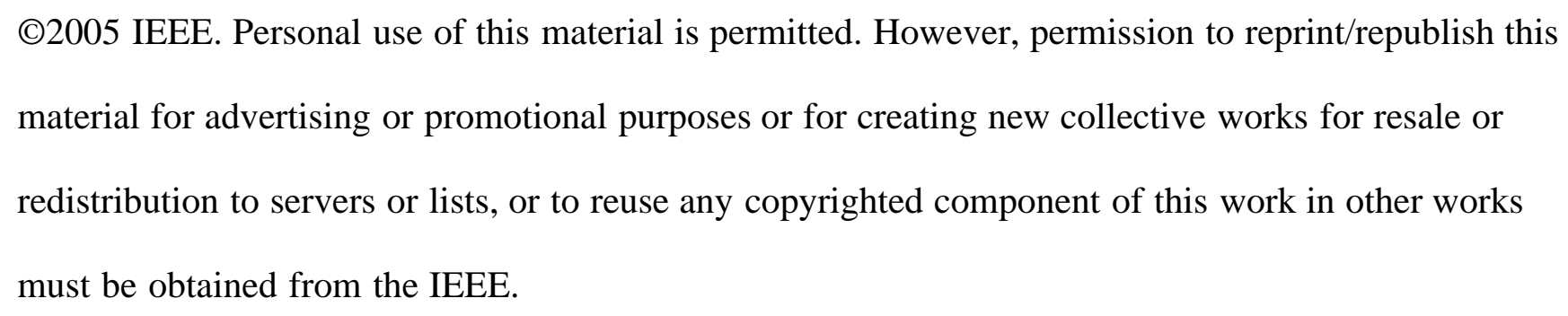




\title{
Robust Linear Receivers for Space-Time Block Coded Multiaccess MIMO Systems With Imperfect Channel State Information
}

\author{
Yue Rong, Student Member, IEEE, Shahram Shahbazpanahi, Member, IEEE, and \\ Alex B. Gershman, Senior Member, IEEE
}

\begin{abstract}
Recently, several linear receiver algorithms have been developed for space-time block-coded multiaccess multiple-input multiple-output (MIMO) wireless communication systems. All these techniques are based on the assumption that the channel state information (CSI) at the receiver side is perfect. However, in practical situations, the available CSI may be imperfect because of channel estimation errors and/or outdated training.

In this paper, we develop new robust linear receiver techniques for joint space-time decoding and interference rejection in multiaccess MIMO systems that use orthogonal space-time block codes and erroneous CSI. The proposed receivers are based on worstcase performance optimization. They are shown to provide a substantially improved robustness against CSI mismatches as compared with the existing linear multiaccess MIMO receivers.
\end{abstract}

Index Terms-Imperfect channel state information, multiaccess MIMO communications, orthogonal space-time block codes, robust linear receivers.

\section{INTRODUCTION}

$\mathbf{S}$ PACE-TIME coding has recently emerged as a powerful approach to exploit spatial diversity and combat fading in multiple-input multiple-output (MIMO) wireless communication systems [1]-[3]. Orthogonal space-time block codes (OSTBCs) [2], [3] represent an attractive class of space-time coding techniques because they enjoy full diversity and low decoding complexity. In the point-to-point MIMO communication case, the optimal maximum likelihood (ML) detector for this class of codes consists of a simple linear receiver that maximizes the

Manuscript received April 19, 2004; revised November 1, 2004. This work was supported in part by the Wolfgang Paul Award Program of the Alexander von Humboldt Foundation and German Ministry of Education and Research; Premier's Research Excellence Award Program of the Ministry of Energy, Science and Technology (MEST) of Ontario; the Discovery Grants Program of the Natural Sciences and Engineering Research Council (NSERC) of Canada; and the Research Partnerships Program of Communications and Information Technology Ontario (CITO). The results of this paper were presented in part at ICASSP'04, Montreal, QC, Canada, May 2004. The associate editor coordinating the review of this manuscript and approving it for publication was Dr. Carlos H. Muravchik.

Y. Rong is with the Department of Communication Systems, University of Duisburg-Essen, D-47057 Duisburg, Germany (e-mail: yue.rong@ieee.org).

S. Shahbazpanahi is with the Department of Electrical and Computer Engineering, McMaster University Hamilton, ON L8S 4K1, Canada (e-mail: shahbaz@mail.ece.mcmaster.ca).

A. B. Gershman is with the Department of Communication Systems, University of Duisburg-Essen, D-47057 Duisburg, Germany, on leave from the Department of Electrical and Computer Engineering, McMaster University, Hamilton, ON L8S 4K1, Canada (e-mail: gershman@ieee.org).

Digital Object Identifier 10.1109/TSP.2005.851199 output signal-to-noise ratio (SNR) and the symbol-by-symbol detector. For each symbol, this ML detector can be interpreted as a matched filter (MF) receiver [4].

In the multiaccess MIMO case, the ML receiver has much more complicated structure and prohibitively high complexity as compared with the ML receiver for the point-to-point MIMO case. Therefore, in multiaccess scenarios, suboptimal but simple linear receivers can be a good choice.

Several linear receiver techniques have been recently developed for space-time coded multiaccess MIMO systems [5]-[8]. For example, a minimum variance (MV) linear receiver has been developed in [5] for direct sequence (DS) code-division multiaccess (CDMA) systems that use multiple antennas and spacetime block coding. However, the scheme proposed in [5] is restricted by transmitters that consist of two antennas only. The latter restriction is dictated by the Alamouti's OSTBC scheme that is adopted in [5].

Another linear technique has been proposed in [6], where a decorrelator receiver has been developed for a DS-CDMA based communication system. This receiver also uses the Alamouti's code and is limited by the assumption that the transmitter consists of two antennas and that not more than two antennas are used at the receiver. Another restriction of the receiver of [6] is that it is applicable only to the binary phase shift keying (BPSK) signal case.

One more linear receiver technique for the multiaccess MIMO case has been proposed in [7]. Similar to [5] and [6], the approach of [7] is restricted to the case of Alamouti's code. Another restriction of this approach is that it cannot suppress more than one interferer.

A more general class of MV linear receivers have been recently proposed in [8]. In contrast to [5]-[7], the techniques of [8] are applicable to the general case of arbitrary OSTBCs and multiple interferers.

A common shortcoming of the techniques in [5]-[8] is that they use the assumption that the exact channel state information (CSI) is available at the receiver. In practice, this condition can be violated because of channel estimation errors that are caused by limited/outdated training as well as the effects of multiaccess interference (MAI) and noise.

In this paper, we develop new robust beamforming-type linear receiver techniques for joint space-time decoding and interference rejection. The proposed receivers can be viewed as robust generalizations of the MV techniques of [8] and are based on the 
worst-case performance optimization approach, which was successfully used to develop robust techniques for adaptive beamforming [9]-[12], multiuser detection [13]-[15], and power control [16], [17]. The proposed worst-case optimization-based receivers are shown to provide an improved robustness in the case of imperfect (erroneous) receiver CSI.

The reminder of this paper is organized as follows. In Section II, the background of the point-to-point and multiaccess flat fading MIMO models is given, and an overview of the MV linear receivers of [8] is presented. Section III develops the new robust MV receivers based on worst-case performance optimization. Simulation results are presented in Section IV, and conclusions are drawn in Section V.

\section{BACKGROUND}

\section{A. Point-to-Point MIMO Model}

The relationship between the input and the output of a singleaccess (point-to-point) MIMO system with $N$ transmit and $M$ receive antennas and flat block-fading channel can be expressed as [3]

$$
\mathbf{Y}=\mathbf{X H}+\mathbf{V}
$$

where

$$
\begin{aligned}
& \mathbf{Y} \triangleq\left[\begin{array}{lll}
\mathbf{y}^{T}(1) & \cdots & \mathbf{y}^{T}(T)
\end{array}\right]^{T} \\
& \mathbf{X} \triangleq\left[\begin{array}{lll}
\mathbf{x}^{T}(1) & \cdots & \mathbf{x}^{T}(T)
\end{array}\right]^{T} \\
& \mathbf{V} \triangleq\left[\begin{array}{lll}
\mathbf{v}^{T}(1) & \cdots & \mathbf{v}^{T}(T)
\end{array}\right]^{T}
\end{aligned}
$$

are the matrices of the received signals, transmitted signals, and noise, respectively, $\mathbf{H}$ is the $N \times M$ complex channel matrix, $T$ is the block length, and $(\cdot)^{T}$ denotes the transpose. Here, $\mathbf{y}(t)=\left[\begin{array}{lll}y_{1}(t) & \cdots & y_{M}(t)\end{array}\right], \mathbf{x}(t)=\left[\begin{array}{llll}x_{1}(t) & \cdots & x_{N}(t)\end{array}\right]$, and $\mathbf{v}(t)=\left[\begin{array}{lll}v_{1}(t) & \cdots & v_{M}(t)\end{array}\right]$ are the complex row vectors of the received signal, transmitted signal, and noise, respectively.

Let us denote complex information-bearing symbols prior to space-time encoding as $s_{1}, s_{2}, \ldots, s_{K}$ and assume that these symbols belong to (possibly different) constellations $\mathcal{U}_{k}, k=$ $1,2, \ldots, K$. Let $\mathbf{s} \triangleq\left[\begin{array}{lll}s_{1} & \cdots & s_{K}\end{array}\right]^{T}$. Note that $\mathbf{s} \in \mathcal{S}$, where $\mathcal{S}=\left\{\mathbf{s}^{(1)} \ldots \mathbf{s}^{(L)}\right\}$ is the set of all possible symbol vectors, and $L$ is the cardinality of this set. The $T \times N$ matrix $\mathbf{X}(\mathbf{s})$ is called an OSTBC if [3]

- all elements of $\mathbf{X}(\mathbf{s})$ are linear functions of the $K$ complex variables $s_{1}, s_{2}, \ldots, s_{K}$ and their complex conjugates;

- for any arbitrary s, it satisfies

$$
\mathbf{X}^{H}(\mathbf{s}) \mathbf{X}(\mathbf{s})=\|\mathbf{s}\|^{2} \mathbf{I}_{N}
$$

where $\mathbf{I}_{N}$ is the $N \times N$ identity matrix, and $\|\cdot\|$ denotes the Euclidean norm of a vector or the Frobenius norm of a matrix.

It can be readily verified that the matrix $\mathbf{X}(\mathbf{s})$ can be written as [8], [18], [19]

$$
\mathbf{X}(\mathbf{s})=\sum_{k=1}^{K}\left(\mathbf{C}_{k} \operatorname{Re}\left\{s_{k}\right\}+\mathbf{D}_{k} \operatorname{Im}\left\{s_{k}\right\}\right)
$$

where $\mathbf{C}_{k} \triangleq \mathbf{X}\left(\tilde{\mathbf{e}}_{k}\right), \mathbf{D}_{k} \triangleq \mathbf{X}\left(j \tilde{\mathbf{e}}_{k}\right), j=\sqrt{-1}$, and $\tilde{\mathbf{e}}_{k}$ is the $K \times 1$ vector having one in the $k$ th position and zeros elsewhere. Using (3), one can rewrite (1) as [8], [18], [19]

$$
\underline{\mathbf{Y}}=\mathbf{A}(\mathbf{H}) \underline{\mathbf{s}}+\underline{\mathbf{V}}
$$

where the "underline" operator for any matrix $\mathbf{P}$ is defined as

$$
\underline{\mathbf{P}} \triangleq\left[\begin{array}{l}
\operatorname{vec}\{\operatorname{Re}(\mathbf{P})\} \\
\operatorname{vec}\{\operatorname{Im}(\mathbf{P})\}
\end{array}\right]
$$

and $\operatorname{vec}\{\cdot\}$ is the vectorization operator stacking all columns of a matrix on top of each other. Here, the $2 M T \times 2 K$ real matrix $\mathbf{A}(\mathbf{H})$ is defined as [8], [19]

$$
\mathbf{A}(\mathbf{H}) \triangleq\left[\begin{array}{llllll}
\underline{\mathbf{C}_{1} \mathbf{H}} & \cdots & \underline{\mathbf{C}_{K} \mathbf{H}} & \underline{\mathbf{D}_{1} \mathbf{H}} & \cdots & \underline{\mathbf{D}_{K} \mathbf{H}}
\end{array}\right] .
$$

The matrix A captures both the effects of the space-time code and the channel. An important property of this matrix is that its columns have the same norms and are orthogonal to each other:

$$
\mathbf{A}^{T}(\mathbf{H}) \mathbf{A}(\mathbf{H})=\|\mathbf{H}\|^{2} \mathbf{I}_{2 K} .
$$

In the presence of the exact CSI at the receiver, the optimal (ML) space-time decoder uses channel knowledge to find the closest point to the received signal in the noise-free observation space $\mathcal{Y}=\left\{\mathbf{Y}^{(1)}, \mathbf{Y}^{(2)}, \ldots, \mathbf{Y}^{(L)}\right\}$, i.e., it obtains [3]

$$
l_{\text {opt }}=\arg \min _{l \in\{1, \ldots, L\}}\left\|\mathbf{Y}-\mathbf{Y}^{(l)}\right\|
$$

and then uses this index to decode the transmitted bits. Here, $\mathbf{Y}^{(l)}$ is the noise-free received signal matrix that corresponds to the vector of information-bearing symbols $\mathbf{s}^{(l)}$.

The ML receiver can also be viewed as a matched filter whose output SNR is maximized [4]. It can be shown [8], [19] that (8) is equivalent to the MF linear receiver, which computes the following estimate of $\underline{\mathbf{s}}$ :

$$
\underline{\hat{\mathbf{s}}}=\frac{1}{\|\mathbf{H}\|^{2}} \mathbf{A}^{T}(\mathbf{H}) \underline{\mathbf{Y}}
$$

and builds the estimate of the vector $\mathbf{s}$ as

$$
\hat{\mathbf{s}}=\left[\begin{array}{ll}
\mathbf{I}_{K} & j \mathbf{I}_{K}
\end{array}\right] \underline{\hat{\mathbf{s}}} .
$$

The $k$ th element of $\hat{\mathbf{s}}$ is then compared with all points in $\mathcal{U}_{k}$. The closest point is accepted as an estimate of $k$ th entry of $\mathbf{s}$. This procedure is repeated for all $k=1,2, \ldots, K$, that is, the decoding is done symbol-by-symbol.

According to (7), the matrix $\left(1 /\|\mathbf{H}\|^{2}\right) \mathbf{A}^{T}(\mathbf{H})$ in (9) is the pseudoinverse of $\mathbf{A}(\mathbf{H})$. Therefore, (9) can be alternatively viewed as a decorrelator receiver.

\section{B. Multiaccess MIMO Model}

Let us now consider an uplink multiaccess MIMO communication system shown in Fig. 1. The transmitters (users) are assumed to have the same number of transmitting antennas 


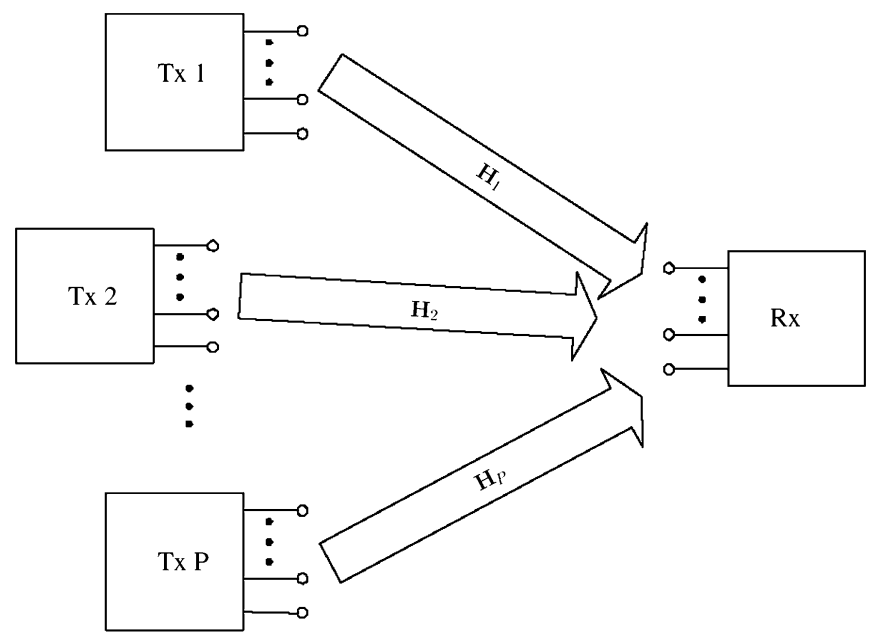

Fig. 1. Multiaccess MIMO system.

and to encode the information-bearing symbols using the same OSTBC. ${ }^{1}$ The received signal is given by [8]

$$
\mathbf{Y}=\sum_{p=1}^{P} \mathbf{X}_{p} \mathbf{H}_{p}+\mathbf{V}
$$

where $\mathbf{X}_{p}$ is the matrix of transmitted signals of the $p$ th transmitter, $\mathbf{H}_{p}$ is the channel matrix between the $p$ th transmitter and the receiver, and $P$ is the number of transmitters.

Applying the "underline" operator of (5) to (11), we have [8]

$$
\underline{\mathbf{Y}}=\sum_{p=1}^{P} \mathbf{A}\left(\mathbf{H}_{p}\right) \underline{\mathbf{s}_{p}}+\underline{\mathbf{V}}
$$

where $\mathbf{s}_{p}$ is a $K \times 1$ vector of information-bearing symbols of the $p$ th transmitter and, according to (6), the matrix $\mathbf{A}\left(\mathbf{H}_{p}\right)$ can be expressed as

$$
\begin{aligned}
\mathbf{A}\left(\mathbf{H}_{p}\right) & =\left[\begin{array}{lllll}
\frac{\mathbf{C}_{1} \mathbf{H}_{p}}{\triangleq} & \cdots & \frac{\mathbf{C}_{K} \mathbf{H}_{p}}{\mathbf{D}_{1} \mathbf{H}_{p}} & \cdots & \frac{\mathbf{D}_{K} \mathbf{H}_{p}}{]}
\end{array}\right] \\
& \triangleq\left[\begin{array}{llll}
\mathbf{a}_{1}\left(\mathbf{H}_{p}\right) & \cdots & \mathbf{a}_{2 K}\left(\mathbf{H}_{p}\right)
\end{array}\right] .
\end{aligned}
$$

In (13), $\mathbf{a}_{k}\left(\mathbf{H}_{p}\right)$ represents the space-time signature that corresponds to the $k$ th real-valued symbol transmitted by the $p$ th user (i.e., the space-time signature that corresponds to the $k$ th component of the vector $\mathbf{s}_{p}$ ). Both the effects of the space-time code and the channel of the $p$ th user are captured in the matrix (13).

\section{Minimum Variance Linear Receivers}

In the multiaccess MIMO case, the MF receiver of (8) becomes highly nonoptimal because it ignores the effect of MAI treating it as a noise. In this case, the receiver performance is determined by the signal-to-interference-plus-noise ratio (SINR) rather than the SNR, and some cancellation of MAI is required.

Using the model (12) and assuming without any loss of generality that the first transmitter is the transmitter-of-interest, we can express the output vector of a linear receiver as [8]

$$
\underline{\hat{\mathbf{s}}_{1}}=\mathbf{W}^{T} \underline{\mathbf{Y}}
$$

${ }^{1}$ These assumptions are only needed for notational simplicity and can be relaxed; see [8]. where

$$
\mathbf{W}=\left[\begin{array}{llll}
\mathbf{w}_{1} & \mathbf{w}_{2} & \cdots & \mathbf{w}_{2 K}
\end{array}\right]
$$

is the $2 M T \times 2 K$ real matrix of the receiver coefficients, and $\hat{\mathbf{s}}_{1}$ is the estimate of the vector $\mathbf{s}_{1}$ at the receiver output. The vector $\mathbf{w}_{k}$ can be interpreted as the weight vector for the $k$ th entry of $\underline{\mathbf{s}_{1}}$.

Given the matrix $\mathbf{W}$, the estimate of the vector of information-bearing symbols of the transmitter-of-interest can be computed as

$$
\hat{\mathbf{s}}_{1}=\left[\begin{array}{ll}
\mathbf{I}_{K} & j \mathbf{I}_{K}
\end{array}\right] \underline{\hat{\mathbf{s}}_{1}} .
$$

Using such a linear estimate, the $k$ th information-bearing symbol can be detected as a point in $\mathcal{U}_{k}$, which is the nearest neighbor to the $k$ th entry of $\hat{\mathbf{s}}_{1}$.

Using the framework of (14), we can interpret the MF receiver in (9) as a linear receiver with the following coefficient matrix:

$$
\mathbf{W}_{\mathrm{MF}}=\frac{1}{\left\|\mathbf{H}_{1}\right\|^{2}} \mathbf{A}\left(\mathbf{H}_{1}\right) .
$$

The similarity of the vectorized multiaccess MIMO model (12) and models used in array processing gives an opportunity to design the matrix $\mathbf{W}$ using the MV principle [20]. Using the MV approach, in [8], it has been proposed to estimate each entry of $\mathbf{s}_{1}$ by minimizing the receiver output power while preserving a unity gain for this particular entry of $\underline{\mathbf{s}_{1}}$, that is

$$
\min _{\mathbf{w}_{k}} \mathbf{w}_{k}^{T} \hat{\mathbf{R}} \mathbf{w}_{k} \quad \text { subject to } \quad \mathbf{a}_{k}^{T}\left(\mathbf{H}_{1}\right) \mathbf{w}_{k}=1
$$

for all $k=1, \ldots, 2 K$, where

$$
\hat{\mathbf{R}}=\frac{1}{J} \sum_{i=1}^{J} \underline{\mathbf{Y}}_{i} \underline{\mathbf{Y}}^{T}
$$

is the sample estimate of the $2 M T \times 2 M T$ full-rank covariance matrix

$$
\mathbf{R} \triangleq \mathrm{E}\left\{\underline{\mathbf{Y}} \underline{\mathbf{Y}}^{T}\right\}
$$

of the vectorized data (12), $\mathbf{Y}_{i}$ is the $i$ th received data block, $J$ is the number of data blocks available, and $\mathrm{E}\{\cdot\}$ denotes the statistical expectation.

The solution to (16) is given by [8]

$$
\mathbf{w}_{\mathrm{MV}, k}=\frac{1}{\mathbf{a}_{k}^{T}\left(\mathbf{H}_{1}\right) \hat{\mathbf{R}}^{-1} \mathbf{a}_{k}\left(\mathbf{H}_{1}\right)} \hat{\mathbf{R}}^{-1} \mathbf{a}_{k}\left(\mathbf{H}_{1}\right)
$$

with $k=1, \ldots, 2 K$. The form of the obtained MV receiver (17) is similar to that of the minimum variance distortionless response (MVDR) receiver used in beamforming [20] and the minimum output energy (MOE) receiver used in multiuser detection [21]. Although the receiver (17) is able to reject MAI, it does not fully cancel self-interference [5], which, for each $\mathbf{w}_{\mathrm{MV}, k}$, is caused by other entries of $\mathbf{s}_{1}$ than the $k$ th one. Note that the complete cancellation of self-interference is a very desirable feature because, otherwise, the symbol-by-symbol detector becomes nonoptimal [8]. 
To incorporate the self-interference cancellation feature into (16), it was proposed in [8] to use additional zero-forcing constraints $\mathbf{a}_{l}^{T}\left(\mathbf{H}_{1}\right) \mathbf{w}_{k}=0$ for all $l \neq k$. These constraints guarantee that self-interference is completely rejected.

With such additional constraints, the problem (16) can be reformulated as [8]

$$
\min _{\mathbf{W}} \operatorname{tr}\left\{\mathbf{W}^{T} \hat{\mathbf{R}} \mathbf{W}\right\} \quad \text { subject to } \quad \mathbf{A}^{T}\left(\mathbf{H}_{1}\right) \mathbf{W}=\mathbf{I}_{2 K}
$$

where $\operatorname{tr}\{\cdot\}$ denotes the trace of a matrix. Using the Lagrange multiplier method, the solution to (18) can be written in the following form [8]:

$$
\tilde{\mathbf{W}}_{\mathrm{MV}}=\hat{\mathbf{R}}^{-1} \mathbf{A}\left(\mathbf{H}_{1}\right)\left[\mathbf{A}\left(\mathbf{H}_{1}\right)^{T} \hat{\mathbf{R}}^{-1} \mathbf{A}\left(\mathbf{H}_{1}\right)\right]^{-1} .
$$

Note that the linear receivers (17) and (19) can be used not only in the case of multiaccess MIMO systems with OSTBCs but in a more general case as well, where linear (not necessarily orthogonal) STBCs are used.

To improve the performance in the case of imperfect CSI and sample size, it was proposed in [8] to apply fixed diagonal loading (DL) to (17) and (19). Then, the DL-based modification of the MV receiver (17) can be written as

$$
\mathbf{W}_{\mathrm{DLMV}}=\left[\begin{array}{lll}
\mathbf{w}_{\mathrm{DLMV}, 1} & \cdots & \mathbf{w}_{\mathrm{DLMV}, 2 K}
\end{array}\right]
$$

with

$$
\mathbf{w}_{\mathrm{DLMV}, k}=\frac{1}{\mathbf{a}_{k}^{T}\left(\mathbf{H}_{1}\right) \hat{\mathbf{R}}_{\mathrm{DL}}^{-1} \mathbf{a}_{k}\left(\mathbf{H}_{1}\right)} \hat{\mathbf{R}}_{\mathrm{DL}}^{-1} \mathbf{a}_{k}\left(\mathbf{H}_{1}\right)
$$

where $k=1, \ldots, 2 K ; \hat{\mathbf{R}}_{\mathrm{DL}} \triangleq \hat{\mathbf{R}}+\gamma \mathbf{I}_{2 M T}$ is the diagonally loaded sample covariance matrix, and $\gamma$ is the fixed DL factor.

Similarly, the DL-based modification of the MV receiver (19) takes the form

$$
\tilde{\mathbf{W}}_{\mathrm{DLMV}}=\hat{\mathbf{R}}_{\mathrm{DL}}^{-1} \mathbf{A}\left(\mathbf{H}_{1}\right)\left[\mathbf{A}\left(\mathbf{H}_{1}\right)^{T} \hat{\mathbf{R}}_{\mathrm{DL}}^{-1} \mathbf{A}\left(\mathbf{H}_{1}\right)\right]^{-1} .
$$

Simulation results in [8] have demonstrated that the receiver (22) usually outperforms (20). Unfortunately, it is not clear from [8] what the proper choice of $\gamma$ in (21) and (22) is and how it depends on the norm of the CSI errors. Furthermore, it is well known that the optimal choice of the DL factor is scenario dependent [9], [15]. Therefore, the robustness of the fixed DL receivers (20) and (22) may be insufficient.

\section{Robust Minimum Variance ReCEIVERS}

In this section, we develop generalizations of the techniques (17) and (19), which are robust against imperfect channel knowledge at the receiver. Let us assume that the true channel matrix $\mathbf{H}_{1}$ is not available at the receiver. The only available quantity is its estimate $\hat{\mathbf{H}}_{1}$, which represents a distorted (mismatched) copy of $\mathbf{H}_{1}$. Let us introduce the error matrix

$$
\boldsymbol{\Delta}_{1} \triangleq \mathbf{H}_{1}-\hat{\mathbf{H}}_{1}
$$

between the true channel matrix $\mathbf{H}_{1}$ and its presumed (estimated) value $\hat{\mathbf{H}}_{1}$, and let the Frobenius norm of this error matrix be upper bounded by a known constant $\varepsilon$, that is

$$
\left\|\boldsymbol{\Delta}_{1}\right\| \leq \varepsilon
$$

Let us define the mismatched space-time signatures $\mathbf{a}_{k}\left(\hat{\mathbf{H}}_{1}\right)(k=1, \ldots, 2 K)$ of the desired user through the matrix

$$
\begin{aligned}
\mathbf{A}\left(\hat{\mathbf{H}}_{1}\right) & =\left[\begin{array}{lllll}
\mathbf{C}_{1} \hat{\mathbf{H}}_{1} & \cdots & \frac{\mathbf{C}_{K} \hat{\mathbf{H}}_{1}}{\mathbf{D}_{1} \hat{\mathbf{H}}_{1}} & \cdots & \underline{\mathbf{D}_{K} \hat{\mathbf{H}}_{1}}
\end{array}\right] \\
& \triangleq\left[\begin{array}{llll}
\mathbf{a}_{1}\left(\hat{\mathbf{H}}_{1}\right) & \cdots & \left.\mathbf{a}_{2 K}\left(\hat{\mathbf{H}}_{1}\right)\right] .
\end{array}\right.
\end{aligned}
$$

The following Lemma will be needed to derive our robust MV receivers.

\section{Lemma 1: For any OSTBC}

$$
\left\|\boldsymbol{\Delta}_{1}\right\|=\left\|\mathbf{e}_{k, 1}\right\| \text { for all } k=1, \ldots, 2 K
$$

where

$$
\mathbf{e}_{k, 1} \triangleq \mathbf{a}_{k}\left(\mathbf{H}_{1}\right)-\mathbf{a}_{k}\left(\hat{\mathbf{H}}_{1}\right) .
$$

Proof: See Appendix A.

The sought robust modification of (16) should minimize the output power subject to the constraint that the distortionless response is maintained for the set of mismatched real-valued space-time signature vectors

$$
\mathcal{A}(\varepsilon)=\left\{\mathbf{c}_{k} \mid \mathbf{c}_{k}=\mathbf{a}_{k}\left(\hat{\mathbf{H}}_{1}+\boldsymbol{\Delta}\right),\|\boldsymbol{\Delta}\| \leq \varepsilon\right\} .
$$

This formulation corresponds to the spherical uncertainty set case [9]. Then, the robust modification of (16) can be written as the following optimization problem:

$$
\min _{\mathbf{w}_{k}} \mathbf{w}_{k}^{T} \hat{\mathbf{R}} \mathbf{w}_{k} \quad \text { subject to } \min _{\|\boldsymbol{\Delta}\| \leq \varepsilon} \mathbf{w}_{k}^{T} \mathbf{a}_{k}\left(\hat{\mathbf{H}}_{1}+\boldsymbol{\Delta}\right) \geq 1
$$

for all $k=1, \ldots, 2 K$. The main modification of (28) with respect to (16) is that for each $k$, instead of requiring fixed distortionless response toward the single mismatched space-time signature $\mathbf{a}_{k}\left(\hat{\mathbf{H}}_{1}\right)$, in (28), such distortionless response is maintained by means of inequality constraints for a continuum of all space-time signatures given by the set $\mathcal{A}(\varepsilon)$. If (24) is satisfied, then from (28), it follows that the distortionless response is also maintained for the true space-time signature $\mathbf{a}_{k}\left(\mathbf{H}_{1}\right)=$ $\mathbf{a}_{k}\left(\hat{\mathbf{H}}_{1}+\boldsymbol{\Delta}_{1}\right)$. The constraints in (28) guarantee that the distortionless response will be maintained in the worst case, i.e., for the particular vector $\mathbf{c}_{k} \in \mathcal{A}\{\varepsilon\}$, which corresponds to the smallest value of $\mathbf{w}_{k}^{T} \mathbf{c}_{k}$.

Using Lemma 1, this problem can be transformed to

$$
\min _{\mathbf{w}_{k}} \mathbf{w}_{k}^{T} \hat{\mathbf{R}} \mathbf{w}_{k} \quad \text { subject to } \min _{\left\|\mathbf{e}_{k}\right\| \leq \varepsilon} \mathbf{w}_{k}^{T}\left(\mathbf{a}_{k}\left(\hat{\mathbf{H}}_{1}\right)+\mathbf{e}_{k}\right) \geq 1
$$


for all $k=1, \ldots, 2 K$. The problem mathematically similar to (29) arises in adaptive beamforming [9], [12] and multiuser detection [15]. Using the results of [9], it can be straightforwardly shown that in the case of a moderate mismatch parameter $\varepsilon$ (i.e., in the case when $\left.\mathbf{w}_{k}^{T} \mathbf{a}_{k}\left(\hat{\mathbf{H}}_{1}\right)>\varepsilon\left\|\mathbf{w}_{k}\right\|\right)$, this problem is equivalent to

$$
\min _{\mathbf{w}_{k}} \mathbf{w}_{k}^{T} \hat{\mathbf{R}} \mathbf{w}_{k} \quad \text { subject to } \mathbf{w}_{k}^{T} \mathbf{a}_{k}\left(\hat{\mathbf{H}}_{1}\right)-\varepsilon\left\|\mathbf{w}_{k}\right\|=1
$$

for all $k=1, \ldots, 2 K$. Applying the Lagrange multiplier method to (30), for each $k=1, \ldots, 2 K$, we obtain that the solution to (30) is given by the equation

$$
2 \hat{\mathbf{R}} \mathbf{w}_{k}+\mu \varepsilon \frac{\mathbf{w}_{k}}{\left\|\mathbf{w}_{k}\right\|}=\mu \mathbf{a}_{k}\left(\hat{\mathbf{H}}_{1}\right)
$$

where $\mu$ is the unknown Lagrange multiplier. To get around the problem of computing $\mu$, let us assume that constant modulus symbol constellations are used. Hence, the vector $\mathbf{w}_{k}$ can be rescaled by an arbitrary constant without affecting the receiver performance [15]. Using this fact and rescaling $\mathbf{w}_{k}$ as $\mathbf{w}_{k}:=$ $\mathbf{w}_{k} / \mu$, we can rewrite (31) as

$$
\mathbf{w}_{k}=\left(2 \hat{\mathbf{R}}+\frac{\varepsilon}{\left\|\mathbf{w}_{k}\right\|} \mathbf{I}_{2 M T}\right)^{-1} \mathbf{a}_{k}\left(\hat{\mathbf{H}}_{1}\right) .
$$

Note that the term $\varepsilon /\left\|\mathbf{w}_{k}\right\|$ can be interpreted as an adaptive diagonal loading factor, which is optimally matched to the given level $\varepsilon$ of the channel uncertainty. To solve (32), we can apply a technique similar to that developed in [15]. From (32), we obtain that the optimal value of $\left\|\mathbf{w}_{k}\right\|$ can be found as the root of the following nonlinear equation:

$$
\sum_{i=1}^{2 M T}\left(\frac{\left[\tilde{\mathbf{a}}_{k}\left(\hat{\mathbf{H}}_{1}\right)\right]_{i}}{2 \lambda_{i}\left\|\mathbf{w}_{k}\right\|+\varepsilon}\right)^{2}=1
$$

where

$$
\hat{\mathbf{R}}=\mathbf{U} \boldsymbol{\Lambda} \mathbf{U}^{T}
$$

is the eigenvalue decomposition of $\hat{\mathbf{R}}$

$$
\boldsymbol{\Lambda}=\operatorname{diag}\left\{\begin{array}{llll}
\lambda_{1} & \lambda_{2} & \cdots & \lambda_{2 M T}
\end{array}\right\}
$$

is the diagonal matrix of the eigenvalues of $\hat{\mathbf{R}}$,

$$
\tilde{\mathbf{a}}_{k}\left(\hat{\mathbf{H}}_{1}\right)=\mathbf{U}^{T} \mathbf{a}_{k}\left(\hat{\mathbf{H}}_{1}\right)
$$

and $[\cdot]_{i}$ denotes the $i$ th element of a vector.

Standard methods such as Newton-Raphson technique can be applied to solve (33); see [15] for more details. Once this equation is solved, the obtained value of $\left\|\mathbf{w}_{k}\right\|$ can be inserted into the right-hand side of (32) to compute the optimal vector $\mathbf{w}_{k}$. Repeating this procedure for all $k=1, \ldots, 2 K$, we obtain the optimal weight matrix $\mathbf{W}$, which is the solution of (28).

Next, let us develop a robust modification of the receiver (19). To obtain such a modification, we should add worst-case zeroforcing constraints for self-interference. Following this idea and taking into account that, in this case, it is impossible to reject self-interference completely, we add to (29) additional constraints to limit the contribution of self-interference to the re- ceiver output power. Then, for each $k$, our problem takes the following form:

$$
\begin{array}{r}
\min _{\mathbf{w}_{k}} \mathbf{w}_{k}^{T} \hat{\mathbf{R}} \mathbf{w}_{k} \quad \text { subject to } \min _{\left\|\mathbf{e}_{k}\right\| \leq \varepsilon} \mathbf{w}_{k}^{T}\left(\mathbf{a}_{k}\left(\hat{\mathbf{H}}_{1}\right)+\mathbf{e}_{k}\right) \geq 1 \\
\max _{\left\|\mathbf{E}_{k}\right\| \leq \eta}\left\|\left(\mathbf{B}_{k}^{T}+\mathbf{E}_{k}^{T}\right) \mathbf{w}_{k}\right\| \leq \delta_{k}
\end{array}
$$

where the $2 M T \times(2 K-1)$ matrices $\mathbf{B}_{k}$ and $\mathbf{E}_{k}$ are defined as

$$
\begin{aligned}
& \mathbf{B}_{k} \triangleq\left[\begin{array}{llllll}
\mathbf{a}_{1}\left(\hat{\mathbf{H}}_{1}\right) & \cdots & \mathbf{a}_{k-1}\left(\hat{\mathbf{H}}_{1}\right) & \mathbf{a}_{k+1}\left(\hat{\mathbf{H}}_{1}\right) & \cdots & \mathbf{a}_{2 K}\left(\hat{\mathbf{H}}_{1}\right)
\end{array}\right] \\
& \mathbf{E}_{k} \triangleq\left[\begin{array}{llllll}
\mathbf{e}_{1} & \cdots & \mathbf{e}_{k-1} & \mathbf{e}_{k+1} & \cdots & \mathbf{e}_{2 K}
\end{array}\right]
\end{aligned}
$$

respectively, $\delta_{k}$ is the value that limits the contribution of selfinterference in the uncertainty region $\left\|\mathbf{E}_{k}\right\| \leq \eta$, and $\eta$ is the upper bound for $\left\|\mathbf{E}_{k}\right\|$.

Lemma 2: For any OSTBC

$$
\eta=\varepsilon \sqrt{2 K-1}
$$

Proof: See Appendix B.

Using triangle and Cauchy-Schwartz inequalities along with $\left\|\mathbf{E}_{k}\right\| \leq \eta$ and Lemma 2, we have

$$
\begin{aligned}
\left\|\left(\mathbf{B}_{k}^{T}+\mathbf{E}_{k}^{T}\right) \mathbf{w}_{k}\right\| & \leq\left\|\mathbf{B}_{k}^{T} \mathbf{w}_{k}\right\|+\left\|\mathbf{E}_{k}^{T} \mathbf{w}_{k}\right\| \\
& \leq\left\|\mathbf{B}_{k}^{T} \mathbf{w}_{k}\right\|+\left\|\mathbf{E}_{k}\right\|\left\|\mathbf{w}_{k}\right\| \\
& \leq\left\|\mathbf{B}_{k}^{T} \mathbf{w}_{k}\right\|+\eta\left\|\mathbf{w}_{k}\right\| \\
& =\left\|\mathbf{B}_{k}^{T} \mathbf{w}_{k}\right\|+\varepsilon \sqrt{2 K-1}\left\|\mathbf{w}_{k}\right\| .
\end{aligned}
$$

It can be readily verified that all the inequalities in (37) become equalities if

$$
\mathbf{E}_{k}=\frac{\eta \mathbf{w}_{k} \mathbf{w}_{k}^{T} \mathbf{B}_{k}}{\left\|\mathbf{w}_{k}\right\|\left\|\mathbf{B}_{k}^{T} \mathbf{w}_{k}\right\|} .
$$

Using the latter observation and (37), we have that

$$
\max _{\left\|\mathbf{E}_{k}\right\| \leq \eta}\left\|\left(\mathbf{B}_{k}^{T}+\mathbf{E}_{k}^{T}\right) \mathbf{w}_{k}\right\|=\left\|\mathbf{B}_{k}^{T} \mathbf{w}_{k}\right\|+\varepsilon \sqrt{2 K-1}\left\|\mathbf{w}_{k}\right\| .
$$

Note that to zero-force self-interference in the uncertainty region as much as possible, the parameter $\delta_{k}$ in (35) should be chosen as small as possible (subject to the constraint that this problem remains feasible). The problem of potential infeasibility, and correspondingly, the problem of choice of $\delta_{k}$ can be avoided by treating $\delta_{k}$ as a variable to be minimized. Following this idea, let us add $\delta_{k}$ to the objective function in (35). In addition, let us use (39) to simplify the second constraint in (35). Then, we obtain the following problem:

$$
\begin{aligned}
\min _{\mathbf{w}_{k}, \delta_{k}} & \sqrt{\mathbf{w}_{k}^{T} \hat{\mathbf{R}} \mathbf{w}_{k}}+\delta_{k} \\
\text { subject to } & \mathbf{w}_{k}^{T} \mathbf{a}_{k}\left(\hat{\mathbf{H}}_{1}\right)-\varepsilon\left\|\mathbf{w}_{k}\right\| \geq 1 \\
& \left\|\mathbf{B}_{k}^{T} \mathbf{w}_{k}\right\|+\varepsilon \sqrt{2 K-1}\left\|\mathbf{w}_{k}\right\| \leq \delta_{k} .
\end{aligned}
$$

Now, let us convert this problem to the convex second-order cone programming (SOCP) form [22]. The canonical SOCP problem has the following formulation [22]:

$$
\begin{array}{r}
\min _{\mathbf{z}} \mathbf{f}^{T} \mathbf{z} \quad \text { subject to }\left\|\mathbf{Q}_{i} \mathbf{z}+\mathbf{b}_{i}\right\| \leq \mathbf{c}_{i}^{T} \mathbf{z}+d_{i} \\
i=1, \ldots, I
\end{array}
$$


where $\mathbf{z} \in \mathbb{R}^{n}$ are the optimization variables, and $\mathbf{f} \in \mathbb{R}^{n}, \mathbf{Q}_{i} \in$ $\mathbb{R}^{\left(n_{i}-1\right) \times n}, \mathbf{b}_{i} \in \mathbb{R}^{n_{i}-1}, \mathbf{c}_{i} \in \mathbb{R}^{n}$, and $d_{i} \in \mathbb{R}$ are the problem parameters. Here, $\mathbb{R}$ is the set of all real numbers. The constraint

$$
\left\|\mathbf{Q}_{i} \mathbf{z}+\mathbf{b}_{i}\right\| \leq \mathbf{c}_{i}^{T} \mathbf{z}+d_{i}
$$

is known as the second-order cone (SOC) of dimension $n_{i}$.

To convert the optimization problem (40) to the canonical SOCP problem, we now use the Cholesky decomposition of $\hat{\mathbf{R}}$ :

$$
\hat{\mathbf{R}}=\mathbf{L}^{T} \mathbf{L}
$$

where $\mathbf{L}$ is an upper-triangular matrix. Using (42), we obtain that

$$
\sqrt{\mathbf{w}_{k}^{T} \hat{\mathbf{R}} \mathbf{w}_{k}}=\sqrt{\mathbf{w}_{k}^{T} \mathbf{L}^{T} \mathbf{L} \mathbf{w}_{k}}=\left\|\mathbf{L} \mathbf{w}_{k}\right\|
$$

Making use of (43) and introducing a new auxiliary variable $\tau_{1}$, which satisfies the inequality $\tau_{1} \geq\left\|\mathbf{L w}_{k}\right\|$, the optimization problem (40) can be equivalently rewritten as

$$
\begin{aligned}
\min _{\tau_{1}, \delta_{k}, \mathbf{w}_{k}} \tau_{1}+\delta_{k} \text { subject to } & \left\|\mathbf{L} \mathbf{w}_{k}\right\| \leq \tau_{1} \\
& \varepsilon\left\|\mathbf{w}_{k}\right\| \leq \mathbf{w}_{k}^{T} \mathbf{a}_{k}\left(\hat{\mathbf{H}}_{1}\right)-1 \\
& \left\|\mathbf{B}_{k}^{T} \mathbf{w}_{k}\right\|+\varepsilon \sqrt{2 K-1}\left\|\mathbf{w}_{k}\right\| \leq \delta_{k} .
\end{aligned}
$$

The first and the second constraints in (44) are already written in the SOCP form. Let us now convert the third constraint of (44) into SOCP constraints. Introducing auxiliary variables $\tau_{2}$ and $\tau_{3}$, this constraint can be written as

$$
\begin{aligned}
\left\|\mathbf{B}_{k}^{T} \mathbf{w}_{k}\right\| & \leq \tau_{2} \\
\varepsilon \sqrt{2 K-1}\left\|\mathbf{w}_{k}\right\| & \leq \tau_{3} \\
\tau_{2}+\tau_{3} & \leq \delta_{k} .
\end{aligned}
$$

Replacing the third constraint in (44) with (45), we finally obtain the following equivalent form of the problem (35):

$$
\begin{aligned}
\min _{\boldsymbol{\tau}, \delta_{k}, \mathbf{w}_{k}} \tau_{1}+\delta_{k} \text { subject to } & \left\|\mathbf{L} \mathbf{w}_{k}\right\| \leq \tau_{1} \\
& \varepsilon\left\|\mathbf{w}_{k}\right\| \leq \mathbf{w}_{k}^{T} \mathbf{a}_{k}\left(\hat{\mathbf{H}}_{1}\right)-1 \\
& \left\|\mathbf{B}_{k}^{T} \mathbf{w}_{k}\right\| \leq \tau_{2} \\
& \varepsilon \sqrt{2 K-1}\left\|\mathbf{w}_{k}\right\| \leq \tau_{3} \\
& \tau_{2}+\tau_{3} \leq \delta_{k}
\end{aligned}
$$

where $\tau=\left[\tau_{1}, \tau_{2}, \tau_{3}\right]^{T}$. Comparing (46) with the canonical form of a SOCP problem given in (41), one can easily see that if $\mathbf{z}=\left[\mathbf{w}_{k}^{T}, \boldsymbol{\tau}^{T}, \delta_{k}\right]^{T}$, then we have five SOCs with $\mathbf{Q}_{1}=\left[\begin{array}{ll}\mathbf{L} & \mathbf{0}_{2 M T \times 4}\end{array}\right], \mathbf{b}_{1}=\mathbf{0}_{2 M T \times 1}, \mathbf{c}_{1}=\left[\begin{array}{lll}\mathbf{0}_{1 \times 2 M T} & 1 & \mathbf{0}_{1 \times 3}\end{array}\right]^{T}$, $d_{1}=0 ; \mathbf{Q}_{2}=\left[\begin{array}{ll}\varepsilon \mathbf{I}_{2 M T} & \mathbf{0}_{2 M T \times 4}\end{array}\right], \mathbf{b}_{2}=\mathbf{0}_{2 M T \times 1}, \mathbf{c}_{2}=$ $\left[\begin{array}{ll}\mathbf{a}_{k}^{T}\left(\hat{\mathbf{H}}_{1}\right) & \mathbf{0}_{1 \times 4}\end{array}\right]^{T}, d_{2}=-1 ; \mathbf{Q}_{3}=\left[\begin{array}{ll}\mathbf{B}_{k}^{T} & \mathbf{0}_{(2 K-1) \times 4}\end{array}\right], \mathbf{b}_{3}=$ $\mathbf{0}_{(2 K-1) \times 1}, \mathbf{c}_{3}=\left[\begin{array}{lll}\mathbf{0}_{1 \times(2 M T+1)} & 1 & \mathbf{0}_{1 \times 2}\end{array}\right]^{T}, d_{3}=0$; $\mathbf{Q}_{4}=\left[\begin{array}{ll}\varepsilon \sqrt{2 K-1} \mathbf{I}_{2 M T} & \mathbf{0}_{2 M T \times 4}\end{array}\right], \mathbf{b}_{4}=\mathbf{0}_{2 M T \times 1}, \mathbf{c}_{4}=$ $\left[\begin{array}{lll}\mathbf{0}_{1 \times(2 M T+2)} & 1 & 0\end{array}\right]^{T}, d_{4}=0 ; \mathbf{Q}_{5}=\mathbf{0}_{1 \times(2 M T+4)}, \mathbf{b}_{5}=0$, $\mathbf{c}_{5}=\left[\begin{array}{lllll}\mathbf{0}_{1 \times(2 M T)} & 0 & -1 & -1 & 1\end{array}\right]^{T}$, and $d_{5}=0$. Here, $\mathbf{0}_{i \times l}$ denotes an $i \times l$ matrix with all zero entries.

Problem (46) represents a convex SOCP problem that can be straightforwardly and efficiently solved using interior point algorithms [22], [23].

Note that (46) can be solved for each value of $k(k=$ $1, \ldots, 2 K)$ independently. In addition, in contrast to our first robust receiver (32), the receiver (46) is not restricted by constant modulus symbol constellations.

It is also worth noting that the proposed receivers (32) and (46) do not need any knowledge of the channel matrices of interfering users.

\section{A. Computational Complexity}

The main computational cost of our first receiver (32) is determined by the matrix inversion and eigendecomposition operations in (32) and (34), respectively. Therefore, the complexity of this receiver is $\mathcal{O}\left(M^{3} T^{3}\right)$. The complexity of our second receiver (46) is mainly determined by the complexity of the corresponding interior point algorithm used to solve the SOCP problem (46) and is equal to $O\left(M^{3} T^{3}\right)$ per iteration [22]. Typically, less than ten to 15 iterations are required to converge (a commonly accepted fact in the optimization community [9], which is also gained by our extensive simulations).

Summarizing, our second receiver may have slightly higher computational complexity than the first one and also requires a specific built-in convex optimization software. This moderate increase in the implementational complexity of (46) is compensated by its more general application to nonconstant modulus signal constellations and, as shown in the next section, by remarkable performance improvements over (32).

\section{Simulations}

Throughout the simulations, we assume a single receiver of $M=8$ antennas. The number of transmitters varies in different simulation examples. The interfering transmitter uses the same OSTBC as the transmitter of interest. The interference-to-noise ratio (INR) is equal to $20 \mathrm{~dB}$, and the QPSK modulation scheme is used. All plots are averaged over 1000 independent simulation runs. In each simulation run, the elements of the true channel matrices $\mathbf{H}_{p}$ (for $p=1, \ldots, P$ ) are independently drawn from a complex Gaussian random generator with zero mean and unit variance.

The proposed robust receivers (32) and (46) are compared to the MF receiver (15) and the DLMV receiver (22). Note that the imperfect CSI case is assumed, i.e., all these receivers use the presumed (erroneous) channel matrix $\hat{\mathbf{H}}_{1}$ rather than the true channel matrix $\mathbf{H}_{1}$. In each simulation run, each element of the presumed channel matrix $\hat{\mathbf{H}}_{1}$ is generated by drawing a complex Gaussian random variable with zero mean and the variance $\sigma_{e}^{2}=0.1$ and adding this variable to a corresponding element of the matrix $\mathbf{H}_{1}$. Moreover, the performance of the so-called informed MV receiver is tested and included in all plots. The latter receiver corresponds to the ideal case when (19) is used with the exactly known $\mathbf{H}_{1}$. Obviously, this receiver does 


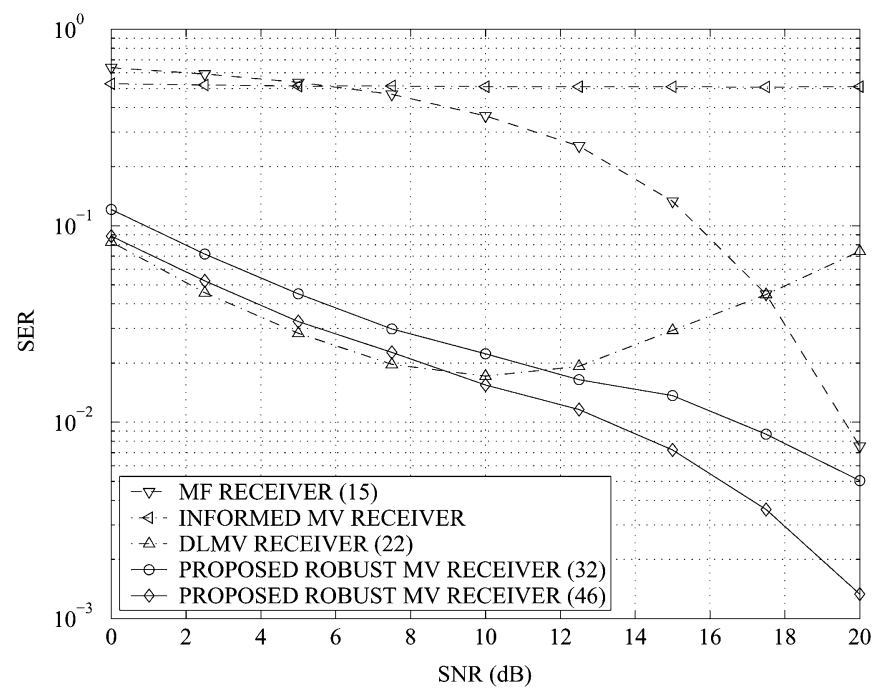

Fig. 2. SER versus SNR; first example. The numbers in the figure legend refer to the equation number of the corresponding receiver in the text.

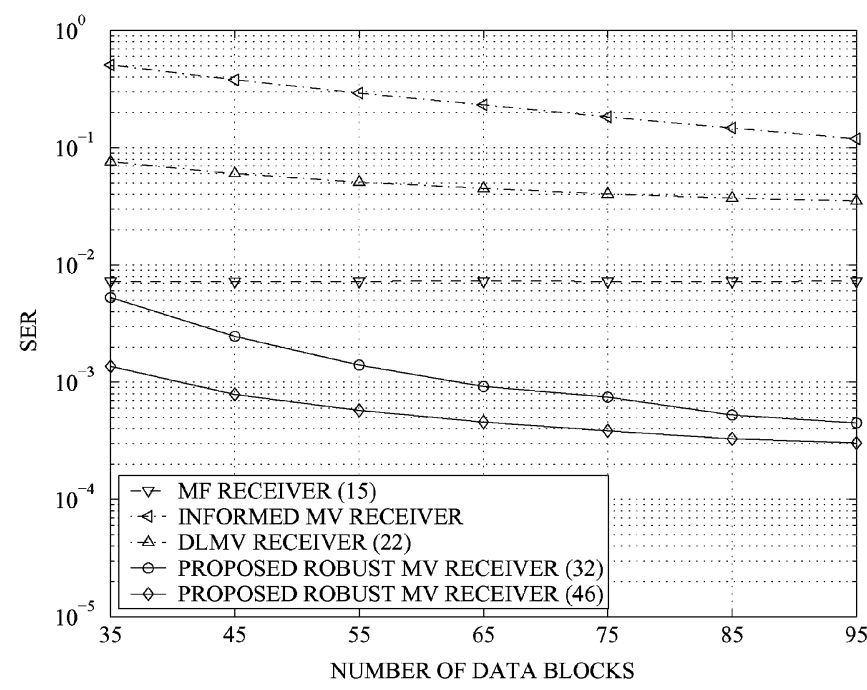

Fig. 3. SER versus number of data blocks; first example. The numbers in the figure legend refer to the equation number of the corresponding receiver in the text.

not correspond to any practical situation and is included in our simulations for the sake of comparison only (as a benchmark).

The SeDuMi convex optimization MATLAB toolbox [23] has been used to solve the SOCP problem (46). The SeDuMi toolbox applies an interior-point method that is computationally efficient. We have observed that the interior-point method typically converges in less than 15 iterations.

The diagonal loading factor of $\gamma=10 \sigma^{2}$ is used in the DLMV receiver, where $\sigma^{2}$ is the noise variance. Note that this is a popular ad hoc choice of $\gamma$ [8], [9].

In the first example, $P=2$ transmitters with $N=2$ antennas per transmitter are assumed, and the full-rate Alamouti's OSTBC ( $T=2, K=2)$ is used [2]. In this example, the parameter $\varepsilon=6 \sigma_{e}$ is used in our robust receivers (32) and (46). It should be noted that this value of $\varepsilon$ is nearly optimal for this example. In Fig. 2, the symbol error rates (SERs) of all the receivers tested are displayed versus the SNR for $J=35$. Fig. 3

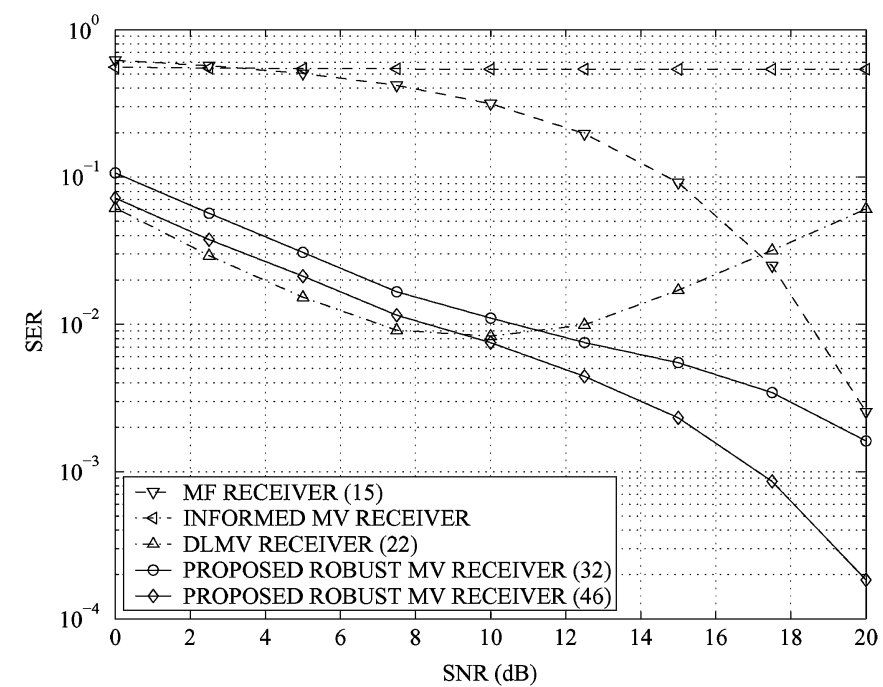

Fig. 4. SER versus SNR; second example. The numbers in the figure legend refer to the equation number of the corresponding receiver in the text.

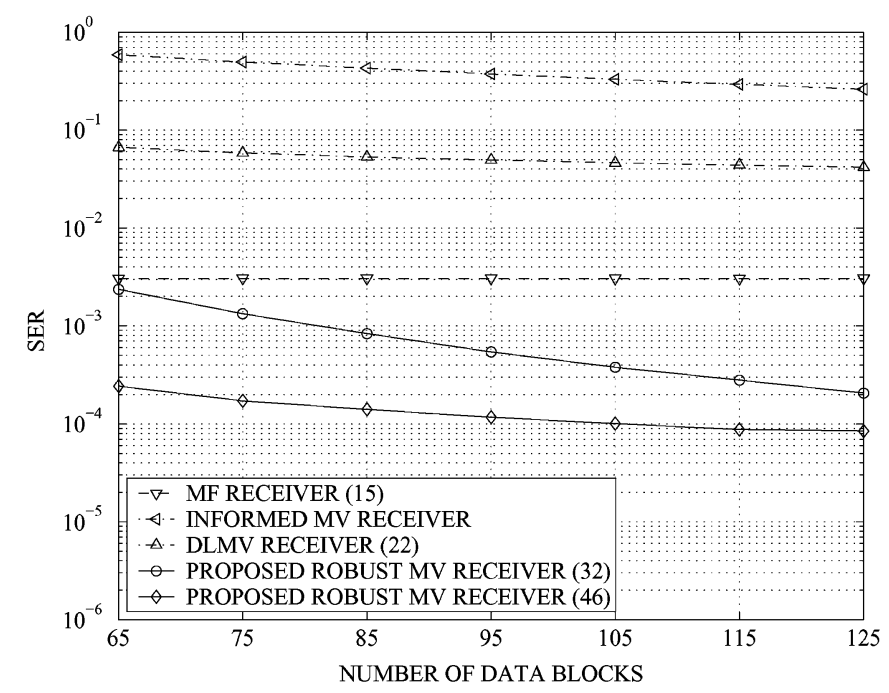

Fig. 5. SER versus number of data blocks; second example. The numbers in the figure legend refer to the equation number of the corresponding receiver in the text.

shows the SERs of the same receivers versus the number of data blocks for $\mathrm{SNR}=20 \mathrm{~dB}$.

In the second example, we assume $P=2$ transmitters. Each transmitter has $N=3$ antennas, and the $3 / 4$-rate $(K=3 ; T=$ 4) orthogonal design STBC from [3] is used. The parameter $\varepsilon=7 \sigma_{e}$ is taken (which is nearly optimal for this example). Fig. 4 shows the receiver SERs versus the SNR for $J=70$, whereas Fig. 5 displays the receiver SERs versus the number of data blocks for SNR $=20 \mathrm{~dB}$.

In the third example, we assume $P=4$ transmitters. Each transmitter has $N=3$ antennas, and the half-rate $(K=4 ; T=$ 8 ) orthogonal design STBC from [3] is used. The parameter $\varepsilon=6 \sigma_{e}$ is taken in this example. Fig. 6 displays the SERs versus SNR of all the receivers tested for $J=130$, whereas Fig. 7 shows the receiver SERs versus the number of data blocks for $\mathrm{SNR}=20 \mathrm{~dB}$.

From Figs. 2-7, it follows that in all examples, the proposed robust receivers (32) and (46) provide better performance 


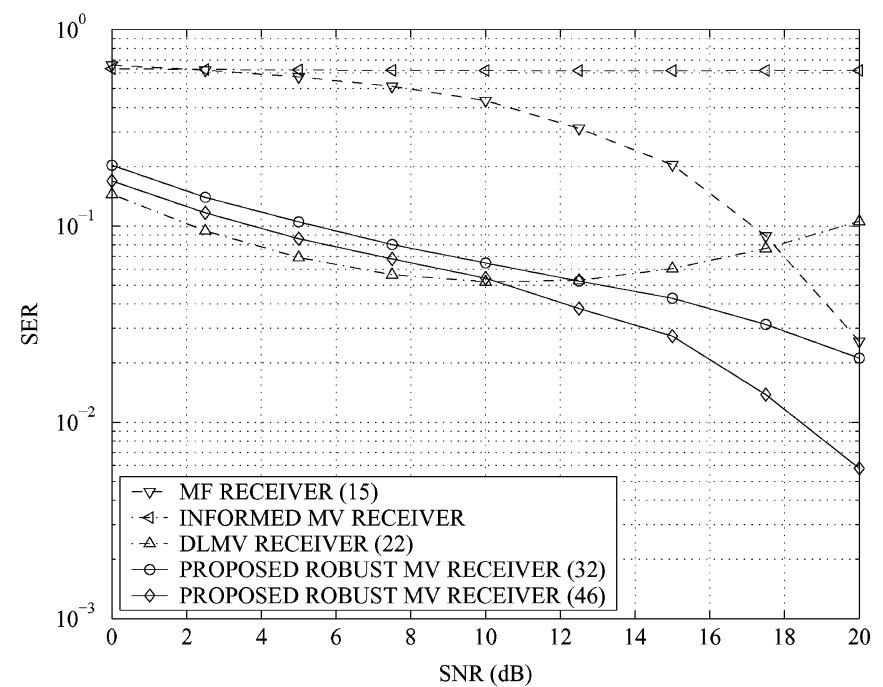

Fig. 6. SER versus SNR; third example. The numbers in the figure legend refer to the equation number of the corresponding receiver in the text.

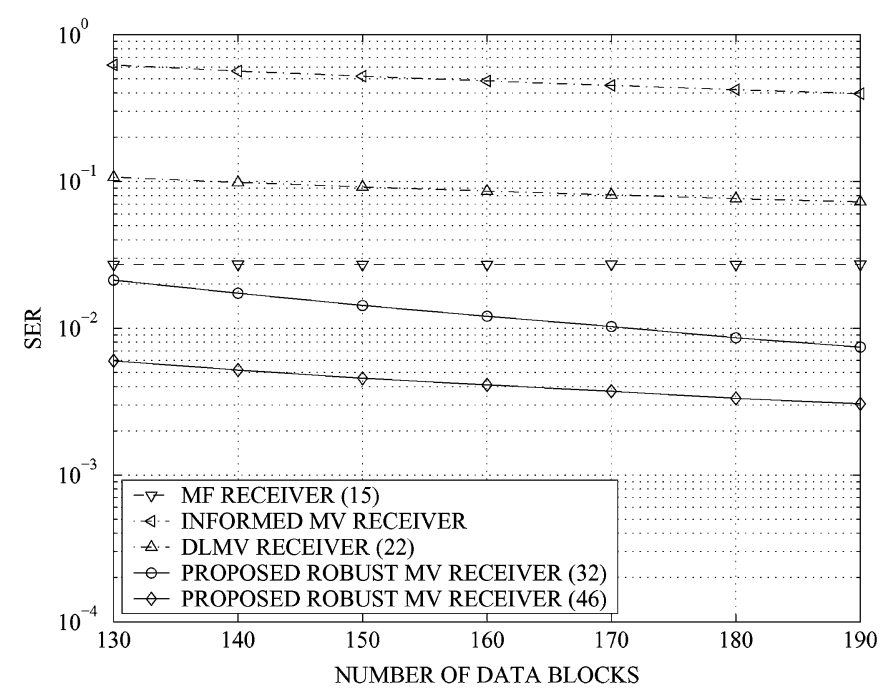

Fig. 7. SER versus number of data blocks; third example. The numbers in the figure legend refer to the equation number of the corresponding receiver in the text.

tradeoffs over the whole tested SNR range as compared to the other receivers (including the informed MV receiver). As expected, the receiver (46) substantially outperforms (32) because the former technique takes advantage of an additional nulling of self-interference; see [8] and the discussion therein. Furthermore, as it can be expected from [8], these performance improvements of (46) relative to (32) are especially pronounced at high SNRs. It can be observed from Figs. 2, 4, and 6 that very substantial performance improvements over the DLMV receiver at high SNR values are achieved in our robust receivers at the price of slightly worse performance at low SNR values (where both the DLMV and our techniques perform quite well). Such performance degradation of the DLMV receiver at high SNR values can be explained by the fact that this receiver uses the fixed diagonal loading factor. The poor performance of the informed MV receiver is due to its insufficient robustness against finite sample effects.

\section{CONCLUSION}

New linear receiver techniques for joint space-time decoding and interference rejection in multiaccess MIMO systems have been developed. These techniques use orthogonal space-time block codes and have an improved robustness against CSI errors. The proposed algorithms are based on worst-case performance optimization and can be implemented in a computationally efficient way. They have been shown to provide a substantially improved robustness against CSI mismatches as compared with the existing nonrobust multiaccess MIMO receiver algorithms.

\section{APPENDIX A}

PROOF OF LEMMA 1

Let

$$
\mathbf{G}_{k} \triangleq \begin{cases}\mathbf{C}_{k}, & k=1, \ldots, K \\ \mathbf{D}_{k-K}, & k=K+1, \ldots, 2 K\end{cases}
$$

Using this notation, from (13), we obtain

$$
\mathbf{a}_{k}\left(\mathbf{H}_{1}\right)=\underline{\mathbf{G}_{k} \mathbf{H}_{1}}, \quad k=1, \ldots, 2 K .
$$

Using the linearity of the underline operator (5), the vector $\mathbf{e}_{k, 1} \triangleq \mathbf{a}_{k}\left(\mathbf{H}_{1}\right)-\mathbf{a}_{k}\left(\hat{\mathbf{H}}_{1}\right)$ can be written as

$$
\mathbf{e}_{k, 1}=\underline{\mathbf{G}_{k} \mathbf{H}_{1}}-\underline{\mathbf{G}_{k} \hat{\mathbf{H}}_{1}}=\underline{\mathbf{G}_{k} \boldsymbol{\Delta}_{1}} .
$$

Using (5) along with well-known properties of the Kronecker product and the vec $\{\cdot\}$ operator [24], (47) can be rewritten as

$$
\begin{aligned}
\mathbf{e}_{k, 1} & =\left[\begin{array}{l}
\operatorname{vec}\left\{\operatorname{Re}\left(\mathbf{G}_{k} \boldsymbol{\Delta}_{1}\right)\right\} \\
\operatorname{vec}\left\{\operatorname{Im}\left(\mathbf{G}_{k} \boldsymbol{\Delta}_{1}\right)\right\}
\end{array}\right] \\
& =\left[\begin{array}{l}
\operatorname{Re}\left(\left(\mathbf{I}_{M} \otimes \mathbf{G}_{k}\right) \operatorname{vec}\left\{\boldsymbol{\Delta}_{1}\right\}\right) \\
\operatorname{Im}\left(\left(\mathbf{I}_{M} \otimes \mathbf{G}_{k}\right) \operatorname{vec}\left\{\boldsymbol{\Delta}_{1}\right\}\right)
\end{array}\right] \\
& =\left[\begin{array}{ll}
\operatorname{Re}\left(\mathbf{I}_{M} \otimes \mathbf{G}_{k}\right) & -\operatorname{Im}\left(\mathbf{I}_{M} \otimes \mathbf{G}_{k}\right) \\
\operatorname{Im}\left(\mathbf{I}_{M} \otimes \mathbf{G}_{k}\right) & \operatorname{Re}\left(\mathbf{I}_{M} \otimes \mathbf{G}_{k}\right)
\end{array}\right]\left[\begin{array}{c}
\operatorname{vec}\left\{\operatorname{Re}\left(\boldsymbol{\Delta}_{1}\right)\right\} \\
\operatorname{vec}\left\{\operatorname{Im}\left(\boldsymbol{\Delta}_{1}\right)\right\}
\end{array}\right] .
\end{aligned}
$$

Using this equation, the Euclidean norm of $\mathbf{e}_{k, 1}$ can be expressed as

$$
\begin{aligned}
\left\|\mathbf{e}_{k, 1}\right\|^{2}=\left[\operatorname{vec}^{T}\left\{\operatorname{Re}\left(\boldsymbol{\Delta}_{1}\right)\right\} \quad \operatorname{vec}^{T}\left\{\operatorname{Im}\left(\boldsymbol{\Delta}_{1}\right)\right\}\right] \\
\cdot\left[\mathbf{I}_{2 M} \otimes\left(\mathbf{G}_{k}^{H} \mathbf{G}_{k}\right)\right]\left[\begin{array}{l}
\operatorname{vec}\left\{\operatorname{Re}\left(\boldsymbol{\Delta}_{1}\right)\right\} \\
\operatorname{vec}\left\{\operatorname{Im}\left(\boldsymbol{\Delta}_{1}\right)\right\}
\end{array}\right] .
\end{aligned}
$$

For any OSTBC, the following property holds [4], [19]:

$$
\mathbf{G}_{k}^{H} \mathbf{G}_{k}=\mathbf{I}_{N} \quad \text { for all } k=1, \ldots, 2 K .
$$

Using (49), (48) can be simplified as

$$
\begin{aligned}
\left\|\mathbf{e}_{k, 1}\right\|^{2}= & \operatorname{vec}^{T}\left\{\operatorname{Re}\left(\boldsymbol{\Delta}_{1}\right)\right\} \operatorname{vec}\left\{\operatorname{Re}\left(\boldsymbol{\Delta}_{1}\right)\right\} \\
& +\operatorname{vec}^{T}\left\{\operatorname{Im}\left(\boldsymbol{\Delta}_{1}\right)\right\} \operatorname{vec}\left\{\operatorname{Im}\left(\boldsymbol{\Delta}_{1}\right)\right\} \\
= & \left\|\boldsymbol{\Delta}_{1}\right\|^{2} .
\end{aligned}
$$

Lemma 1 is proven. 


\section{APPENDIX B \\ PROOF OF LEMMA 2}

Using the definition of the Frobenius norm along with Lemma 1 (i.e., using the constraints $\left\|\mathbf{e}_{k}\right\|^{2} \leq \varepsilon ; k=1, \ldots, 2 K$ ), we obtain

$$
\begin{aligned}
\left\|\mathbf{E}_{k}\right\| & =\sqrt{\operatorname{tr}\left\{\mathbf{E}_{k}^{T} \mathbf{E}_{k}\right\}} \\
& =\sqrt{\sum_{i=1 ; i \neq k}^{2 K} \mathbf{e}_{i}^{T} \mathbf{e}_{i}} \\
& \leq \varepsilon \sqrt{2 K-1}
\end{aligned}
$$

where the inequality in the third row of (51) becomes an equality if and only if $\left\|\mathbf{e}_{k}\right\|^{2}=\varepsilon ; k=1, \ldots, 2 K$. Hence, from (51), we conclude that

$$
\eta=\max \left\|\mathbf{E}_{k}\right\|=\varepsilon \sqrt{2 K-1}
$$

and Lemma 2 is proven.

\section{REFERENCES}

[1] V. Tarokh, N. Seshadri, and A. R. Calderbank, "Space-time codes for high data rate wireless communication: Performance criterion and code construction," IEEE Trans. Inf. Theory, vol. 44, no. 2, pp. 744-765, Mar. 1998.

[2] S. M. Alamouti, "A simple transmit diversity technique for wireless communications," IEEE J. Sel. Areas Commun., vol. 16, no. 8, pp. $1451-1458$, Oct. 1998

[3] V. Tarokh, H. Jafarkhani, and A. R. Calderbank, "Space-time block codes from orthogonal designs," IEEE Trans. Inf. Theory, vol. 45, no. 5, pp. 1456-1467, Jul. 1999.

[4] G. Ganesan and P. Stoica, "Space-time block codes: A maximum SNR approach," IEEE Trans. Inf. Theory, vol. 47, no. 4, pp. 1650-1656, May 2001.

[5] H. Li, X. Lu, and G. B. Giannakis, "Capon multiuser receiver for CDMA systems with space-time coding," IEEE Trans. Signal Process., vol. 50, no. 5, pp. 1193-1204, May 2002.

[6] D. Reynolds, X. Wang, and H. V. Poor, "Blind adaptive space-time multiuser detection with multiple transmitter and receiver antennas," IEEE Trans. Signal Process., vol. 50, no. 6, pp. 1261-1276, Jun. 2002.

[7] A. F. Naguib, N. Seshadri, and A. R. Calderbank, "Applications of spacetime block codes and interference suppression for high capacity and high data rate wireless systems," in Proc. 32nd Asilomar Conf. Signals, Syst., Comput., vol. 2, Pacific Grove, CA, Nov. 1998, pp. 1803-1810.

[8] S. Shahbazpanahi, M. Beheshti, A. B. Gershman, M. GharaviAlkhansari, and K. M. Wong, "Minimum variance linear receivers for multiaccess MIMO wireless systems with space-time block coding," IEEE Trans. Signal Process., vol. 52, no. 12, pp. 3306-3313, Dec. 2004.

[9] S. A. Vorobyov, A. B. Gershman, and Z.-Q. Luo, "Robust adaptive beamforming using worst-case performance optimization: A solution to the signal mismatch problem," IEEE Trans. Signal Process., vol. 51, no. 2, pp. 313-324, Feb. 2003.

[10] S. Shahbazpanahi, A. B. Gershman, Z.-Q. Luo, and K. M. Wong, "Robust adaptive beamforming for general-rank signal models," IEEE Trans. Signal Process., vol. 51, no. 9, pp. 2257-2269, Sept. 2003.

[11] S. Vorobyov, A. B. Gershman, Z.-Q. Luo, and N. Ma, "Adaptive beamforming with joint robustness against mismatched signal steering vector and interference nonstationarity," IEEE Signal Process. Lett., vol. 11, no. 2, pp. 108-111, Feb. 2004.

[12] R. Lorenz and S. Boyd, "Robust minimum variance beamforming," in Proc. 37th Asilomar Conf. Signals, Syst., Comput., Pacific Grove, CA, Nov. 2003, pp. 1345-1352.

[13] S. Cui, Z.-Q. Luo, and Z. Ding, "Robust blind multiuser detection against CDMA signature mismatch," in Proc. ICASSP, Salt Lake City, UT, May 2001, pp. 2297-2300.
[14] S. Shahbazpanahi and A. B. Gershman, "Robust blind multiuser detection for synchronous CDMA systems using worst-case performance optimization," IEEE Trans. Wireless Commun., vol. 3, no. 6, pp. 2232-2245, Nov. 2004.

[15] K. Zarifi, S. Shahbazpanahi, A. B. Gershman, and Z.-Q. Luo, "Robust blind multiuser detection based on the worst-case performance optimization of the MMSE receiver," IEEE Trans. Signal Process., vol. 53, no. 1, pp. 295-305, Jan. 2005.

[16] M. Biguesh, S. Shahbazpanahi, and A. B. Gershman, "Robust power adjustment for transmit beamforming in cellular communication systems," in Proc. ICASSP, vol. 5, Hong Kong, Apr. 2003, pp. 105-108.

[17] — , "Robust downlink power control in wireless cellular systems," EURASIP J. Wireless Commun. Networking, no. 2, pp. 261-272, Dec. 2004. Special Issue on Multiuser MIMO Networks.

[18] B. Hassibi and B. M. Hochwald, "High-rate codes that are linear in space and time," IEEE Trans. Inf. Theory, vol. 48, no. 7, pp. 1804-1824, Jul. 2002.

[19] M. Gharavi-Alkhansari and A. B. Gershman, "Constellation space invariance of orthogonal space-time block codes," IEEE Trans. Inf. Theory, vol. 51, no. 1, pp. 331-334, Jan. 2005.

[20] H. L. Van Trees, Optimum Array Processing: Wiley, 2002.

[21] S. Verdú, Multiuser Detection: Cambridge Univ. Press, 1998.

[22] M. Lobo, L. Vandenberghe, S. Boyd, and H. Lebret, "Application of second-order cone programming," Linear Algebra Applicat., pp. 193-228, Nov. 1998

[23] J. F. Sturm, "Using SeDuMi 1.02, a matlab toolbox for optimization over symmetric cones," Optimiz. Methods Software, vol. 11-12, pp. 625-653, 1999.

[24] J. W. Brewer, "Kronecker products and matrix calculus in system theory," IEEE Trans. Circuits Syst., vol. CAS-25, no. 9, pp. 772-781, Sep. 1978.

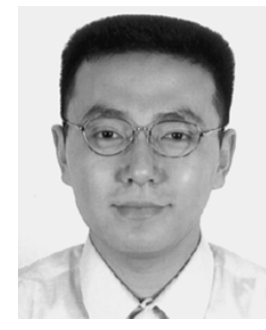

Yue Rong (S'03) was born in 1976 in Jiangsu, China. In 1999, he received the Bachelor degrees from Shanghai Jiao Tong University, Shanghai, China, both in electrical and computer engineering. $\mathrm{He}$ received the M.Sc. degree in computer science and communication engineering from the University of Duisburg-Essen, Duisburg, Germany, in 2002. Currently, he is working toward the Ph.D degree at the Department of Communication Systems, University of Duisburg-Essen.

From April 2001 to April 2002, he was a student research assistant at the Fraunhofer Institute of Microelectronic Circuits and Systems. From October 2001 to March 2002, he was with the Application-Specific Integrated Circuit Design Department, Nokia Ltd., Bochum, Germany. His research interests include signal processing for communications, MIMO communication systems, multicarrier communications, statistical and array signal processing, and parallel factor analysis.

Mr. Rong received the 2001-2002 Graduate Sponsoring Asia scholarship of DAAD/ABB (Germany), and the 2004 Outstanding Ph.D. Student Abroad Award (China).

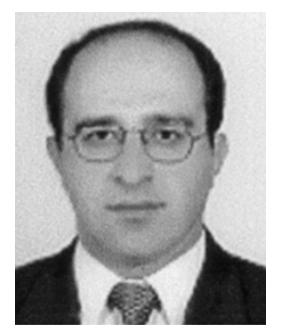

Shahram Shahbazpanahi (M'02) was born in Sanandaj, Kurdistan, Iran. He received the B.Sc., M.Sc., and Ph.D. degrees from Sharif University of Technology, Tehran, Iran, in 1992, 1994, and 2001, respectively, all in electrical engineering.

From September 1994 to September 1996, he was a Faculty Member with the Department of Electrical Engineering, Razi University, Kermanshah, Iran. Since July 2001, he has been conducting research as a Postdoctoral Fellow at the Department of Electrical and Computer Engineering, McMaster University, Hamilton, ON, Canada. From March to September 2002, he was a visiting researcher with the Department of Communication Systems, Gerhard-Mercator University, Duisburg, Germany. His research interests include statistical and array signal processing, space-time adaptive processing, detection and estimation, smart antennas, spread spectrum techniques, and DSP programming and hardware/real-time software design for telecommunication systems. 


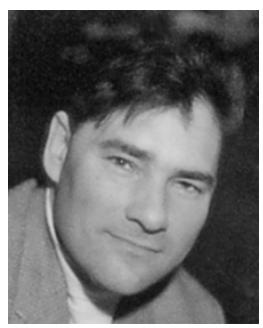

Alex B. Gershman (M'97-SM'98) received the Diploma (M.Sc.) and Ph.D. degrees in radiophysics from the Nizhny Novgorod State University, Nizhny Novgorod, Russia, in 1984 and 1990, respectively.

From 1984 to 1989, he was with the Radiotechnical and Radiophysical Institutes, Nizhny Novgorod. From 1989 to 1997, he was with the Institute of Applied Physics, Russian Academy of Science, Nizhny Novgorod, as a Senior Research Scientist. From the summer of 1994 until the beginning of 1995, he was a Visiting Research Fellow at the Swiss Federal Institute of Technology, Lausanne, Switzerland. From 1995 to 1997, he was Alexander von Humboldt Fellow at Ruhr University, Bochum, Germany. From 1997 to 1999, he was a Research Associate at the Department of Electrical Engineering, Ruhr University. In 1999, he joined the Department of Electrical and Computer Engineering, McMaster University, Hamilton, ON, Canada, where he became a Professor in 2002. From 2002 to 1005 , he held a visiting professorship at the Department of Communication Systems, University of Duisburg-Essen, Duisburg, Germany. Since April 2005, he has been a Professor and Head of the Communications Systems Group at Darmstadt University of Technology, Darmstadt, Germany. His research interests are in the area of signal processing and communications, and include statistical and array signal processing, adaptive beamforming, spatial diversity in wireless communications, multiuser and MIMO communications, parameter estimation and detection, and spectral analysis. He has published over 240 technical papers in these areas.

Dr. Gershman was a recipient of the 1993 International Union of Radio Science (URSI) Young Scientist Award, the 1994 Outstanding Young Scientist Presidential Fellowship (Russia), the 1994 Swiss Academy of Engineering Science and Branco Weiss Fellowships (Switzerland), and the 1995-1996 Alexander von Humboldt Fellowship (Germany). He received the 2000 Premier's Research Excellence Award of Ontario and the 2001 Wolfgang Paul Award from the Alexander von Humboldt Foundation, Germany. He is also a recipient of the 2002 Young Explorers Prize from the Canadian Institute for Advanced Research (CIAR), which has honored Canada's top 20 researchers 40 years of age or under. He co-received the 2004 IEEE Signal Processing Society Best Paper Award. He is an Associate Editor of the IEEE TRANSACTIONS ON Signal PROCESSING, the EURASIP Journal on Wireless Communications and Networking, and the EURASIP Signal Processing Journal and is a Member of both the Sensor Array and Multichannel Signal Processing (SAM) and Signal Processing Theory and Methods (SPTM) Technical Committees of the IEEE Signal Processing Society. He was Technical Co-Chair of the Third IEEE International Symposium on Signal Processing and Information Technology, Darmstadt, Germany, in December 2003. He is Technical Co-Chair of the Fourth IEEE Workshop on Sensor Array and Multichannel Signal Processing, to be held in Waltham, MA, in July 2006. 